\title{
Molecular epidemiology of clinical Mycobacterium tuberculosis complex isolates in South Omo, Southern Ethiopia
}

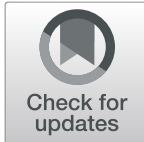

Biniam Wondale ${ }^{1,2^{*}}$ (D) Kwon Keehwan ${ }^{3}$, Girmay Medhin ${ }^{1}$, Takele Teklu ${ }^{1,4}$, Temesgen Mohammed', Samuel Tolosa ${ }^{1}$, Aboma Zewude ${ }^{1,5}$, Friehiwot Amsalu ${ }^{6,7}$, Rembert Pieper $^{3}$ and Gobena Ameni ${ }^{1,8}$

\begin{abstract}
Background: Tuberculosis (TB) is caused by Mycobacterium tuberculosis complex (MTBC). Mapping the genetic diversity of MTBC in high TB burden country like Ethiopia is important to understand principles of the disease transmission and to strengthen the regional TB control program. The aim of this study was to investigate the genetic diversity of Mycobacterium tuberculosis complex (MTBC) isolates circulating in the South Omo, southern Ethiopia.

Methods: MTBC isolates ( $N=156)$ were genetically analyzed using spacer oligotyping (spoligotyping) and mycobacterial interspersed repetitive unit-variable number of tandem repeat (MIRU-VNTR) typing. Major lineages and lineages were identified using MTBC databases. Logistic regression was used to correlate patient characteristics with strain clustering.

Results: The study identified Euro-American (EA), East-African-Indian (EAI), Indo-Oceanic (IO), Lineage_7/ Aethiops vertus, Mycobacterium bovis and Mycobacterium africanum major lineages in proportions of 67.3\% (105/156), 22.4\% (35/156), 6.4\% (10/156), 1.9\% (3/156), 1.3\% (2/156) and 0.6\% (1/156), respectively. Lineages identified were Delhi/CAS 23.9\% (37/155), Ethiopia_2 20.6\% (32/155), Haarlem 14.2\% (22/155), URAL 14.2\%(22/ 155), Ethiopia_3 8.4\% (13/155), TUR 6.5\% (10/155), Lineage_7/Aethiops vertus 1.9\% (3/155), Bovis 1.3\% (2/155), LAM 1.3\% (2/155), EAI 0.6\% (1/155), X 0.6\% (1/155) and Ethiopia $\mathrm{H}_{37}$ Rv-like strain 0.6\% (1/155). Of the genotyped isolates $5.8 \%(9 / 155)$ remained unassigned. The recent transmission index (RTI) was 3.9\%. Orphan strains compared to shared types (AOR: $0.09,95 \% \mathrm{Cl}$ : 0.04-0.25) were associated with reduced odds of clustering. The dominant TB lineage in pastoral areas was EAI and in non-pastoral areas was EA.

Conclusion: The epidemiological data, highly diverse MTBC strains and a low RTI in South Omo, provide information contributing to the TB Control Program of the country.
\end{abstract}

Keywords: M. Tuberculosis, Molecular epidemiology, Spoligotyping, MIRU-VNTR, South Omo

\footnotetext{
* Correspondence: biniamw2005@yahoo.com; biniam.wondale@amu.edu.et

'Aklilu Lemma Institute of Pathobiology, Addis Ababa University, PO. Box

1176, Addis Ababa, Ethiopia

2Department of Biology, Arba Minch University, PO. Box 21, Arba Minch,

Ethiopia

Full list of author information is available at the end of the article
}

(c) The Author(s). 2020 Open Access This article is licensed under a Creative Commons Attribution 4.0 International License, which permits use, sharing, adaptation, distribution and reproduction in any medium or format, as long as you give appropriate credit to the original author(s) and the source, provide a link to the Creative Commons licence, and indicate if changes were made. The images or other third party material in this article are included in the article's Creative Commons licence, unless indicated otherwise in a credit line to the material. If material is not included in the article's Creative Commons licence and your intended use is not permitted by statutory regulation or exceeds the permitted use, you will need to obtain permission directly from the copyright holder. To view a copy of this licence, visit http://creativecommons.org/licenses/by/4.0/ The Creative Commons Public Domain Dedication waiver (http://creativecommons.org/publicdomain/zero/1.0/) applies to the data made available in this article, unless otherwise stated in a credit line to the data. 


\section{Background}

The Mycobacterium tuberculosis complex (MTBC) constitutes a group of mycobacteria which are $99.9 \%$ similar at the nucleotide level and the causative agents for tuberculosis (TB) [11]. Globally, TB became the leading cause of death from an infectious disease [39]. Ethiopia stands 12th in the world and 4th in Africa among the high TB burden countries with 24,000 TB deaths and 165,000 new TB cases in 2018 [39]. The current prevalence of MDR/RR-TB in Ethiopia is 0.71 and $16 \%$ in new and previously treated TB cases, respectively [39].

Understanding the molecular epidemiology of $\mathrm{TB}$ is important for regional disease control. For instance, distinct strains may be linked to outbreaks [10], high virulence [42], emergency of drug resistance [44], disease progression [43], and can point to the geographic origin of a strain $[20,33]$ as well as identify new lineages $[17,28]$.

The South Omo Zone is an administrative unit in the southern Ethiopia bordering Kenya and South Sudan. The area is remote with a poor infrastructure and high population diversity with 16 different ethnic groups. Forty-two percent of South Omo's residents including 15 ethnicities have a pastoral life style. The facilities for health care and education are underdeveloped especially in the pastoral regions [4]. A previous study suggested that the prevalence of TB among pastoralists is higher than in other socio-economic groups in Ethiopia [24]. In depth, high resolution molecular epidemiological surveys are required to characterize the diversity of MTBC isolates in this remote pastoral region precisely.

Beginning in the 1990s a number of molecular genotyping techniques have evolved to differentiate MTBC at the species and strain levels [22]. Whole genome sequencing is ideal to identify a strain type, but technically and informatically demanding and too expensive to characterize regional MTBC diversity [22]. Widely used in TB research are spacer oligotyping (spoligotyping) and mycobacterial interspersed repetitive units - variable numbers of tandem repeat (MIRU-VNTR). Spoligotyping targets a single locus and has less discriminatory power but is simple and cost effective. MIRU-VNTR targets numerous loci with increased discriminatory power. International databases and data analysis tools were created for both genotyping methods [22].

Most MTBC genotyping studies in Ethiopia employed spoligotyping $[3,6,7,16,19,26,43]$. We are aware of only very few Ethiopian studies using spoligotyping and MIRU-VNTR simultaneously to profile MTBC strains $[1,9,36,37,43]$. New lineages such as the MTBC lineage lineage_7/Aethiops vertus [17, 28], Ethiopia_2 and Ethiopia_3 [37] were newly assigned. Most surveys were in geographic areas more accessible than South Omo. The objective of the current study is to examine the MTBC population structures in the latter region and compare the data to those of other Ethiopian regions and globally.

\section{Methods \\ Human subject recruitment and specimen collections}

As part of a study to investigate TB in South Omo using both molecular epidemiology and systems biology methods between 2014 and 2017, we established a human subject protocol and a consent form to recruit more than 2000 individuals from different ethnic groups and administrative districts. We discerned non-pastoral areas (Debub Ari, Semen Ari and part of Male) and pastoral areas (Bena Tsemay, Hamer, Dassenech, Selamago and part of Male). The minimum age for enrolment was 15 years. According to 2007 population census, 577,673 people $(7.5 \%$ urban and $92.5 \%$ rural) lived in the area with equal proportions of men and women [14]. Basic demographic and clinical data were collected using a pre-structured questionnaire.

Local translators explained the study's objectives, risks and benefits to those individuals who were not able to communicate in the Amharic language. Those individuals who consented to participate in the study were asked to provide fine needle aspirate (FNA) and sputum samples to test for EPTB and PTB, respectively. Samples were stored at $+4{ }^{\circ} \mathrm{C}$ and $-20^{\circ} \mathrm{C}$ following collection and transferred to the sample processing and microbial culture laboratory in order to screen for growth of MTBC isolates. Individuals who were diagnosed with $\mathrm{TB}$ were educated on and received DOTS treatment according to the national TB management guideline [18].

\section{Generation of MTBC isolates}

MTBC isolates were recovered from sputum and FNA samples for pulmonary TB (PTB) and extra-pulmonary TB (EPTB) cases, respectively. Mycobacterial cultures were performed at the Jinka Regional Laboratory (JRL). Samples were processed according to the modified Petroff's method by decontaminating the specimen with an equal volume of $4 \% \mathrm{NaOH}$ for $15 \mathrm{~min}$; the remaining volume was filled with phosphate buffer saline (PBS) and centrifuged at speed of $3000 \mathrm{~g}$ for $15 \mathrm{~min}$. A drop of phenol red was added to a pellet as $\mathrm{pH}$ indicator and neutralized using $10 \% \mathrm{HCl}$. The neutralized pellet was inoculated onto two LJ media. One supplemented with glycerol and the other with pyruvate. Inoculated media were incubated at $37^{\circ} \mathrm{C}$ for up to 8 weeks. Mycobacterial growth was monitored every week. Culture was considered negative after 8 weeks if no growth was observed. Positive colonies were further confirmed by Ziehl-Neelsen (ZN) staining. Heat treatment of mycobacterial isolates in $\mathrm{dH}_{2} \mathrm{O}$ at $80{ }^{\circ} \mathrm{C}$ for 50 min was used for genomic DNA extraction without extensive DNA purification. Extracts were stored at $-20{ }^{\circ} \mathrm{C}$ until they were used for molecular characterization. 


\section{Molecular characterization}

DNA of mycobacterial isolates were primarily subjected to region of difference (RD) 9 typing [11]. Secondly, a single tube amplification method [40] was applied for genus typing for isolates which were not detected by RD9 typing. Thirdly, spoligotyping was performed for MTBC isolates as described by Kamerbeek et al., [23]. Laboratory results of spoligotyping were interpreted in binary format and lineages were assigned using an updated version of the SITVITWEB [13] and major lineages were assigned using the "TB insight" database. Isolates which have similar patter to those in the SITV IT database were assigned a Spoligo International Type (SIT) number. Isolates not assigned to SIT numbers were referred as "Orphans" spoligotype. Finally, MTBC DNA samples were subjected to MIRU-VNTR typing following an established procedure [35]. Laboratory results of MIRU-VNTR typing were interpreted using the MIRU-VNTRplus database (http://www.miru-vntrplus. org) to determine MTBC strain lineages and relatedness $[2,38]$. A minimum spanning tree (MST) was constructed. Previously identified Ethiopian strains were assigned manually to Ethiopia_1 (Lineage 7), Ethiopia_2 and Ethiopia_3, which lack the spoligotype based spacer $4-24,13$ and 10-19, respectively [9, 17, 37]. The RD9 typing, genus typing and spoligotyping were performed at Aklilu Lemma Institute of Pathobiology (ALIPB), Addis Ababa University (AAU), Addis Ababa, Ethiopia whereas MIRU-VNTR typing was performed at J. Craig Venter Institute (JCVI), Maryland, USA.

\section{Data analysis}

Excel data were transformed into IBM SPSS version 20 . The results were presented using descriptive statistics. The ability of genotyping methods to discriminate strains was calculated using Hunter-Gaston Discrimination Index (HGDI) [21]. The allelic diversity $(h)$ based on MIRU-VNTR genotyping was calculated using HGDI and classified into relative discriminants based on previous [34] and newly proposed [27] index ranges. The recent transmission index (RTI) was calculated using a formula proposed by small et al. [33]. Isolates were said to be clustered if they have identical pattern based on spoligotyping and/or MIRU-VNTR. Clustering rate was determined using a formula $(\mathrm{Nc}-\mathrm{C}) / \mathrm{N}$, where $\mathrm{Nc}=$ total number of isolates clustered, $\mathrm{C}=$ number of clusters, and $\mathrm{N}=$ total number of isolates [46]. The association between clustered strains and independent variables were computed using logistic regression models. The crude odds ratio (COR) and the adjusted odds ratio (AOR) were used to present the results. $P$-values of $<0.05$ were considered statistically significant.

\section{Results}

\section{Demographic and clinical characteristics of study} participants

One thousand two hundred sputum and FNA samples of study participants were cultured for isolation of mycobacteria. Samples culture summary is presented in Table 1. In overall, $161 \mathrm{MTBC}$ isolates were obtained upon RD9 and multiplex PCR based species and genus typing. Culture recovery rate vary from $2.5 \%(13 / 517)$ in community screened samples and $29.7 \%$ (33/111) in smear positive PTB samples from health facilities other than JGH to $67.3 \%(74 / 110)$ in smear positive PTB samples and 76.5\% (26/34) in EPTB samples from JGH. Spoligotyping and MIRU-VNTR typing were carried out for a total of 156 isolates derived from 130 and 26 patients with clinical evidence of PTB and EPTB, respectively. Five samples were excluded due to insufficient quantities of DNA. Demographic and clinical characteristics of the corresponding 156 subjects are summarized in Table 2. The age range was $15-80$ years with mean 32.9, median 30 and standard deviations 12.9. Female, PTB and new TB cases were 41.0\% (64/156), 83.3\% (130/156) and 95\% (148/156), respectively.

\section{Discriminatory power of genotyping methods and MIRU- VNTR allelic diversity}

All South Omo MTBC isolates were genotyped using spoligotyping as well as MIRU-VNTR at the strain level, as presented in Tables 3 and 4 . The discriminatory power of

Table 1 Culture summary of collected samples, South Omo, Ethiopia

\begin{tabular}{|c|c|c|c|c|c|c|c|}
\hline \multirow{4}{*}{$\begin{array}{l}\text { Source } \\
\text { Sample type }\end{array}$} & \multicolumn{5}{|c|}{ Samples from Health Facilities } & \multirow{4}{*}{$\begin{array}{l}\text { Community }>=2 \text { weeks } \\
\text { cough in pastoral areas } \\
\text { Sputum }\end{array}$} & \multirow[t]{4}{*}{ Total } \\
\hline & \multicolumn{3}{|l|}{ JGH } & \multirow{2}{*}{\multicolumn{2}{|c|}{$\begin{array}{l}\text { Other than JGH } \\
\text { Sputum Smear }\end{array}$}} & & \\
\hline & \multicolumn{2}{|c|}{ Sputum Smear } & \multirow[t]{2}{*}{ FNA } & & & & \\
\hline & + ve & -ve & & $+\mathrm{ve}$ & -ve & & \\
\hline Number of samples & 110 & 404 & 34 & 111 & 24 & 517 & 1200 \\
\hline Recovered MTBC N (\%) & $74(67.3)$ & $14(3.5)$ & $26(76.5)$ & $33(29.7)$ & $1(4.2)$ & $13(2.5)$ & $161(13.4)$ \\
\hline Contamination N (\%) & $4(3.6)$ & $21(5.2)$ & $0(0.0)$ & $6(5.4)$ & $4(16.7)$ & $64(12.4)$ & $99(8.3)$ \\
\hline
\end{tabular}

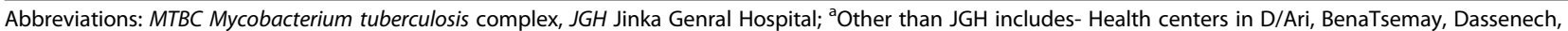
Hammer, Male, Gnagatom and Selamago 
Table 2 Demographic and clinical characteristics of study participants in molecular epidemiology, South Omo, Ethiopia

\begin{tabular}{|c|c|c|c|c|c|c|c|c|c|c|c|}
\hline \multirow[t]{3}{*}{ Characteristics } & \multicolumn{10}{|c|}{ Residential areas } & \multirow{3}{*}{$\begin{array}{l}\text { Total } \\
\text { N (\%) }\end{array}$} \\
\hline & \multicolumn{3}{|c|}{ Non-pastoral $(N=70)$} & \multicolumn{6}{|c|}{ Pastoral $(N=67)$} & \multirow[t]{2}{*}{ Unspecified } & \\
\hline & $\begin{array}{l}\text { D/Ari } \\
N(\%)\end{array}$ & $\begin{array}{l}\text { Jinka } \\
\mathrm{N}(\%)\end{array}$ & $\begin{array}{l}{ }^{\mathrm{a}} \text { Other } \\
\text { areas } \\
\mathrm{N}(\%)\end{array}$ & $\begin{array}{l}\text { B/Tsemay } \\
\mathrm{N}(\%)\end{array}$ & $\begin{array}{l}\text { Dassenech } \\
\mathrm{N}(\%)\end{array}$ & $\begin{array}{l}\text { Hamer } \\
\mathrm{N}(\%)\end{array}$ & $\begin{array}{l}\text { Male } \\
\text { N (\%) }\end{array}$ & $\begin{array}{l}\text { Gnangatom } \\
\mathrm{N}(\%)\end{array}$ & $\begin{array}{l}\text { Selemago } \\
\mathrm{N}(\%)\end{array}$ & & \\
\hline \multicolumn{12}{|l|}{ Sex } \\
\hline Female & $23(48.9)$ & $6(37.5)$ & $1(14.3)$ & $6(42.9)$ & $4(44.4)$ & $4(26.7)$ & $5(41.7)$ & $1(50.0)$ & $7(46.7)$ & $7(36.8)$ & $64(41.0)$ \\
\hline Male & $24(51.1)$ & $10(62.5)$ & $6(85.7)$ & $8(57.1)$ & $5(55.6)$ & $11(73.3)$ & $7(58.3)$ & $1(50.0)$ & $8(53.3)$ & $12(63.2)$ & $92(59.0)$ \\
\hline \multicolumn{12}{|l|}{ Age in years } \\
\hline $15-29$ & $19(40.4)$ & $11(68.8)$ & $2(28.6)$ & $7(50.0)$ & $4(44.4)$ & $2(13.3)$ & $7(58.3)$ & $1(50.0)$ & $11(73.3)$ & $6(31.6)$ & $70(44.9)$ \\
\hline $30-44$ & $19(40.4)$ & $3(18.8)$ & $5(71.4)$ & $3(21.4)$ & $1(11.1)$ & $9(60.0)$ & $2(16.7)$ & $1(50.0)$ & $3(20.0)$ & $13(68.4)$ & $59(37.8)$ \\
\hline $45+$ & $9(19.1)$ & $2(12.5)$ & $0(0.0)$ & $4(28.6)$ & $4(44.4)$ & $4(26.7)$ & $3(25.0)$ & $0(0.0)$ & $1(6.7)$ & $0(0.0)$ & $27(17.3)$ \\
\hline \multicolumn{12}{|l|}{ Type of TB } \\
\hline PTB & 37 (78.7) & $13(81.2)$ & $3(42.9)$ & $13(92.9)$ & $9(100.0)$ & $15(100.0)$ & $10(83.3)$ & $2(100.0)$ & $9(60.0)$ & $19(100.0)$ & $130(83.3)$ \\
\hline EPTB & $10(21.3)$ & $3(18.8)$ & $4(57.1)$ & $1(7.1)$ & $0(0.0)$ & $0(0.0)$ & $2(16.7)$ & $0(0.0)$ & $6(37.5)$ & $0(0.0)$ & $26(16.7)$ \\
\hline \multicolumn{12}{|l|}{ Medical history } \\
\hline New cases & $45(95.7)$ & $16(100.0)$ & $7(100.0)$ & $12(85.7)$ & $9(100.0)$ & $12(80.0)$ & $12(100.0)$ & $2(100.00)$ & $14(93.3)$ & $19(0.0)$ & $148(94.9)$ \\
\hline Retreat. Cases & $2(4.3)$ & $0(0.0)$ & $0(0.0)$ & $2(14.3)$ & $0(0.0)$ & $3(20.0)$ & $0(0.0)$ & $0(0.0)$ & $1(6.7)$ & $0(0.0)$ & $8(5.1)$ \\
\hline Total & $47(30.1)$ & 16 (10.3) & $7(4.5)$ & $14(9.0)$ & $9(5.8)$ & $15(9.6)$ & $12(7.7)$ & $2(1.3)$ & $15(9.6)$ & 19 (12.2) & $156(100.0)$ \\
\hline
\end{tabular}

${ }^{\mathrm{a} O t h e r}$ areas, include Addis Ababa, Konso, Semen Ari and Sawula; D/Ari, Debub Ari; B/Tsemay, Bena Tsemay; Retreat. Cases, anti-TB retreatment cases

spoligotying and 24-loci MIRU-VNTR were 0.9700 and 0.9995 , respectively. The analysis of spoligotypes resulted in 66 different patterns of which 24 clustered and 42 were unique. MIRU-VNTR analysis resulted in 149 different patterns of which six clustered and 143 were unique. The clustering rates were $57.7 \%$ ((114-24)/156) for spoligotying and 3.9\% ((12-6)/155) for the MIRU-VNTR analysis. The RTIs for South Omo were calculated to be $57.7 \%$ based on spoligotyping and 3.9\% based on MIRU-VNTR data.

Allelic diversities $(h)$ for each MIRU-VNTR locus are presented in Table 4. MIRU-VNTR loci are classified based on their allelic diversity and ability to discriminate among the isolates. Eleven MIRU-VNTR loci (424, 577, 802, 960, 1644, 1955, 2163b, 2347, 2996, 3192 and 4052) were highly discriminatory while 10 MIRU-VNTR loci (580, 2059, 2165, 2401, 2461, 2531, 3171, 3690, 4156 and $4348)$ were moderately discriminatory. The remaining three loci showed poor discriminatory values for the isolates under study. However, 11-loci are considered as moderately discriminatory according to newly proposed discriminatory values [27].

\section{Spoligotyping and MIRU-VNTR based identification of lineages and sub-lineages}

The results of spoligotyping and MIRU-VNTR are presented in Tables 5 and 6. According to spoliogotyping results $76.3 \%(119 / 156)$ of the isolates belonged to 36 shared types (SIT numbers). The remaining, about one-qaurter of the isolates (37/156) were orphan strains. Euro-American (EA) was the most prevalent lineage with $67.3 \%$ (105/156) followed by East-African-Indian (EAI) with 22.4\% (35/156) and Indo-Oceanic (IO) with $6.4 \%(10 / 156)$ of the isolates. Interestingly, EA was more prevalent in non-pastoral areas while EAI was more prevalent in pastoral areas (Fig. 1). Two M. bovis isolates were identified in pastoral areas.

According to SITVIT analysis, the identified lineages were $\mathrm{T}$ 30.1\% (47/156), CAS1_Delhi 10.9\% (17/156),

Table 3 Discriminatory power of genotyping methods

\begin{tabular}{|c|c|c|c|c|c|c|c|}
\hline Typing Method & $\begin{array}{l}\text { Different } \\
\text { patterns } \\
\text { (N) }(\%)\end{array}$ & Clusters (N) & $\begin{array}{l}\text { Cluster } \\
\text { size (N) }\end{array}$ & $\begin{array}{l}\text { Clustered } \\
\text { isolates } \\
\text { (N) (\%) }\end{array}$ & $\begin{array}{l}\text { Unique } \\
\text { isolates } \\
\text { (N) (\%) }\end{array}$ & $\begin{array}{l}\text { Clustering } \\
\text { rate } \%\end{array}$ & HGDI \\
\hline Spoligotyping & $66 / 156(42.3)$ & 24 & $2-17$ & $114(73.1)$ & $42(26.9)$ & 57.7 & 0.97 \\
\hline MIRU-VNTR-24 & 149/155 (96.1) & 6 & 2 & $12(7.7)$ & $143(92.3)$ & 3.9 & 0.9995 \\
\hline MIRU-VNTR -15 & $148 / 155$ (95.5) & 7 & 2 & $14(9.0)$ & $141(91.0)$ & 4.5 & 0.9994 \\
\hline MIRU-VNTR-12 & 102/155 (65.8) & 23 & $2-8$ & $76(49.0)$ & $79(51.0)$ & 34.2 & 0.9897 \\
\hline Spoligotyping + MIRU-VNTR & 155/155 (100) & 0 & 0 & 0 & $155(100)$ & 100 & 1 \\
\hline
\end{tabular}


Table 4 Occurrence of MIRU-VNTR alleles and allelic diversity

\begin{tabular}{|c|c|c|c|c|c|c|c|c|c|c|c|c|c|c|c|c|c|c|c|c|}
\hline \multicolumn{3}{|c|}{ MIRU- VNTR } & \multicolumn{16}{|c|}{ Allele No.\# } & \multirow[t]{2}{*}{$h$} & \multirow{2}{*}{$\begin{array}{l}\text { Discriminant } \\
\text { Status }\end{array}$} \\
\hline 24 & 15 & 12 & 0 & 1 & 2 & 3 & 4 & 5 & 6 & 7 & 8 & 9 & 10 & 11 & 12 & 13 & 14 & 15 & & \\
\hline 154 & & & 1 & 7 & 135 & 6 & 2 & 2 & 1 & & & & & & 1 & & & & 0.239 & Poor \\
\hline 424 & & & 10 & 43 & 81 & 14 & 3 & 2 & 1 & 1 & & & & & & & & & 0.641 & High \\
\hline 577 & & & & & 38 & 25 & 84 & 6 & & & & & 1 & & & & 1 & & 0.623 & High \\
\hline 580 & & & & 12 & 129 & 3 & 5 & 1 & 3 & 2 & & & & & & & & & 0.301 & Moderate \\
\hline 802 & & & & 40 & 57 & 52 & 3 & & 1 & 1 & 1 & & & & & & & & 0.690 & High \\
\hline 960 & & & 2 & & 30 & 15 & 46 & 24 & 29 & 4 & 2 & & 2 & 1 & & & & & 0.810 & High \\
\hline 1644 & & & 12 & 35 & 74 & 25 & 6 & 1 & & & & 1 & & & & & & & 0.688 & High \\
\hline 1955 & & & & 2 & 17 & 77 & 54 & & 1 & & & & 1 & & & 2 & 1 & & 0.623 & High \\
\hline 2059 & & & 2 & 17 & 126 & 7 & & & & & & 1 & & & & 1 & & & 0.318 & Moderate \\
\hline $2163 b$ & & & & 20 & 57 & 6 & 30 & 8 & 10 & 15 & 2 & 3 & & 1 & & & 3 & & 0.797 & High \\
\hline 2165 & & & & & 7 & 82 & 56 & 4 & 3 & & 1 & & 1 & & & & & & 0.585 & Moderate \\
\hline 2347 & & & & & 26 & 45 & 79 & 3 & & & & & & & 1 & & & & 0.627 & High \\
\hline 2401 & & & & 2 & 53 & 9 & 90 & 1 & & & & & & & & & & & 0.546 & Moderate \\
\hline 2461 & & & 1 & 7 & 117 & 23 & 5 & 1 & 1 & & & & & & & & & & 0.408 & Moderate \\
\hline 2531 & & & & & & 22 & 11 & 116 & 4 & 1 & & & & & & & & 1 & 0.417 & Moderate \\
\hline 2687 & & & 2 & 137 & 13 & & 1 & & & & 1 & 1 & & & & & & & 0.213 & Poor \\
\hline 2996 & & & & 49 & 1 & 1 & 39 & 37 & 20 & 5 & 3 & & & & & & & & 0.767 & High \\
\hline 3007 & & & & 2 & 9 & 139 & 3 & & & & 1 & & & & 1 & & & & 0.193 & Poor \\
\hline 3171 & & & & 2 & 13 & 115 & 21 & 2 & & & & & & & & & 1 & & 0.419 & Moderate \\
\hline 3192 & & & 1 & 2 & 46 & 76 & 26 & 3 & & & & 1 & & & & & & & 0.647 & High \\
\hline 3690 & & & 2 & 5 & 3 & 103 & 27 & 5 & 6 & 3 & 1 & & & & & & & & 0.527 & Moderate \\
\hline 4052 & & & & 1 & 1 & 9 & 20 & 35 & 24 & 52 & 7 & 3 & 1 & & 1 & & & & 0.792 & High \\
\hline 4156 & & & 4 & 24 & 102 & 6 & 3 & 1 & 2 & 2 & & & 1 & & 1 & 2 & 1 & & 0.505 & Moderate \\
\hline 4348 & & & 2 & 6 & 115 & 28 & & 3 & & 1 & & & & & & & & & 0.418 & Moderate \\
\hline
\end{tabular}

The MIRU-VNTR locus in the Mtb genome; ${ }^{*}$ the number of alleles pertains to the frequency with which a distinct repeat unit was identified among the 155 isolates. High discriminant $(\mathrm{h}>0.6)$, moderate discriminant $(0.3<=\mathrm{h}<=0.6)$ and poor discriminant $(\mathrm{h}<0.3)$ [34]. Shaded boxes show alleles used for the 15-loci MIRU-VNTR and the 12-loci MIRU-VNTR methods

Haarlem 10.3\% (16/156), Ethiopian 1.9\% (3/156), Turkey 1.9 (3/156), MANU 1.3\% (2/156), LAM 0.6\% (1/156), EAI1_SOM 0.6\% (1/156), BOV 0.6\% (1/156) and X $0.6 \%$ (1/156). Almost one-third of isolates (56/156) were not registered in updated version of SITVITWEB database (SITVIT2). According to MIRU-VNTRplus analysis result (S1 Fig, Table 5 and Table 6), the identified lineages were Delhi/CAS 23.9\% (37/155), Ethiopia_2 20.6\% (32/155), Haarlem and URAL 14.2\% (22/155) each, Ethiopia_3 8.4\% (13/155), TUR 6.5\% (10/155), Ethiopia_1 1.9\% (3/155), Bovis and LAM 1.3\% (2/155) each, and EAI, X and Ethiopia $\mathrm{H}_{37} \mathrm{Rv}$-like strain $0.6 \%(1 / 155)$ each. Around $6 \%$ of the isolates were not assigned into lineages using MIRUVNTRplus database.

\section{Minimum spanning tree (MST) analysis}

MST shows the relationship among strains and lineages based on MIRU-VNTR loci variant count. Five welldefined branches (Complex 1, 2, 3, 4 and 5) and four additional sub-branches (Complex 6, 7, 8 and 9) expanding from Complex 1 are featured in Fig. 2. Each complex largely consisted of distinct sub-lineages. Part of Complex 1 are the Ethiopia_2 sub-lineage and the additional sub-lineages H37Rv, Bovis, unidentified strains, and $M$. canetti (used as a reference). Complexes 2, 3, 4 and 5 are enriched in Ethiopian_3 and TUR, URAL, Delhi/ CAS and Haarlem strains, respectively.

\section{Factors associated with strain clustering}

Clustering of $M$. tuberculosis strains association with different factors were analyzed using spoligotyping pattern. Clustering was significantly associated with the residential area, the major lineage type and the SIT status before adjusted to confounding factors (Table 7). However, only being part of the orphan group compared to shared type (AOR: 0.19, 95\% CI: 0.04-0.25) was significantly associated with reduced odds of clustering in the area under study after adjustment with confounding factors.

\section{Discussion}

This study was the first of its kind to analyze the MTBC population structure and transmission dynamics in the South Omo Zone, southern Ethiopia. The study included PTB and EPTB patients, and identified highly diverse lineages. The clustering rate/RTI was low in the study area. Logistic regression analysis showed that clustering of strains was associated with SIT status.

Health facilities other than JGH in the study area are in range of 14 to $250 \mathrm{~km}$ from Jinka town where JGH and the Regional Laboratory are located. Due to feasibility, samples were stored in health facilities at $-20^{\circ} \mathrm{C}$ from 
Table 5 Major lineages and lineages of MTBC strains concordant with existing SIT numbers

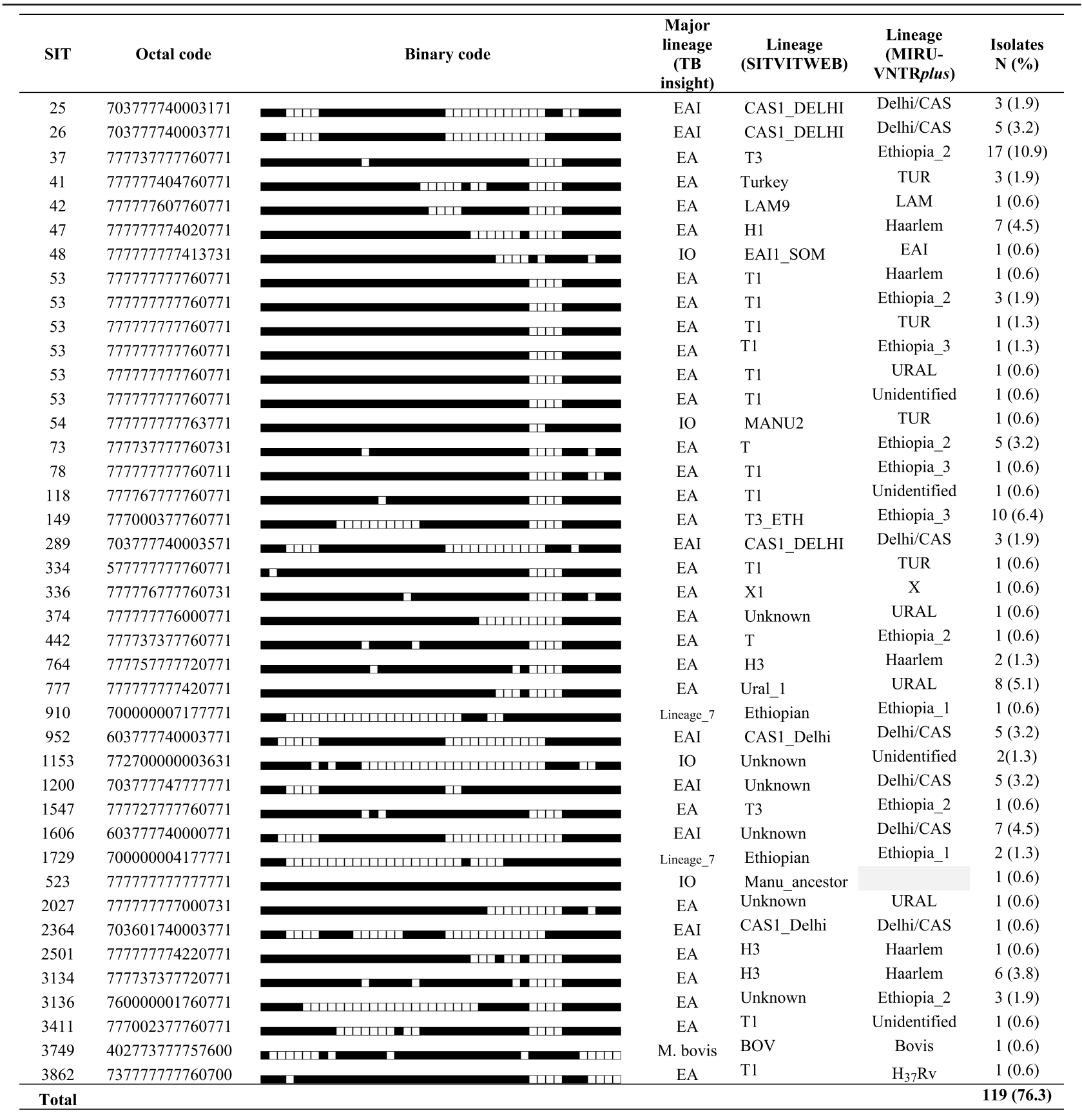

Abbreviations: EA Euro-American, EAI East-African-Indian, $I O$ Indo-oceanic

a week to 3 weeks. The variation in culture recovery rate of MTBC isolates in this study possibly associated with sample storage conditions. There was continuous electric interruption in the Zone which affects the storage temperature which could compromise the viability of MTBC in the sample. JGH had its own backup generator that might be the reason for better recovery rate for samples from JGH. In connection, the overall culture recovery rate in this study is less than previous studies in Ethiopia [47, 48].

In contrast to previous reports in the study area [8, 41], the number of ЕРТВ cases in this study was low. From personal observations, the low number of EPTB cases in this study might be due to lack of skilled pathologist to take FNA samples whereas in previous studies relied on clinical symptoms. Spoligotyping and MIRU- 
Table 6 Major lineages and lineages of orphan MTBC strains

\begin{tabular}{|c|c|c|c|c|c|c|}
\hline SIT & Octal code & Binary code & $\begin{array}{c}\text { Major } \\
\text { lineage } \\
\text { (TB } \\
\text { insight) } \\
\end{array}$ & $\begin{array}{c}\text { Lineage } \\
\text { (SITVITWEB } \\
\text { ) }\end{array}$ & $\begin{array}{c}\text { Lineage } \\
\text { (MIRU- } \\
\text { VNTRplus) }\end{array}$ & $\begin{array}{c}\text { Issolates } \\
\mathbf{N}(\%)\end{array}$ \\
\hline Orphan-1 & 000003437540771 & пाпाпाшпा & EA & Unknown & Unidentified & $1(0.6)$ \\
\hline Orphan-2 & 001777777420731 & $=11110=0$ & $\mathrm{EA}$ & Unknown & URAL & $2(1.3)$ \\
\hline Orphan-3 & 400003435540771 & : & EA & Unknown & URAL & $1(0.6)$ \\
\hline Orphan-4 & 400022237560771 & - & EA & Unknown & Haarlem & $1(0.6)$ \\
\hline Orphan-6 & 400343777776600 & : & M. bovis & Unknown & Bovis & $1(0.6)$ \\
\hline Orphan-7 & 500027437560771 & : & EA & Unknown & Haarlem & $1(0.6)$ \\
\hline Orphan-8 & 501767400142771 & משв" & EAI & Unknown & URAL & $1(0.6)$ \\
\hline Orphan-9 & 501777777423771 & 1110 & IO & Unknown & URAL & $1(0.6)$ \\
\hline Orphan-10 & 540000001740661 & प्याँ & M. afri. & Unknown & Ethiopia_2 & $1(0.6)$ \\
\hline Orphan-11 & 577737377720771 & $-1111=$ & EA & Unknown & Haarlem & $1(0.6)$ \\
\hline Orphan-12 & 577767777400771 & माना & IO & Unknown & Delhi/CAS & $1(0.6)$ \\
\hline Orphan-13 & 603777740000271 & (111 & EAI & Unknown & Delhi/CAS & $1(0.6)$ \\
\hline Orphan-14 & 603777740000671 & 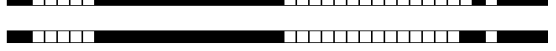 & EAI & Unknown & Delhi/CAS & $1(0.6)$ \\
\hline Orphan-15 & 603777757560771 & 111 & EA & Unknown & Delhi/CAS & $1(0.6)$ \\
\hline Orphan-17 & 703777744203771 & & EAI & Unknown & Delhi/CAS & $2(1.3)$ \\
\hline Orphan-18 & 717777777763771 & 1 & EA & Unknown & Unidentified & $1(0.6)$ \\
\hline Orphan-21 & 763777777563771 & & EA & Unknown & Delhi/CAS & $1(0.6)$ \\
\hline Orphan-24 & 772702000003631 & пाпानाना & IO & Unknown & Unidentified & $2(1.3)$ \\
\hline Orphan-25 & 777002000020771 & משחس سחس & EA & Unknown & Ethiopia_3 & $1(0.6)$ \\
\hline Orphan-27 & 777347777777771 & & IO & Unknown & URAL & $1(0.6)$ \\
\hline Orphan-28 & 777727377720771 & & EA & Unknown & Haarlem & $1(0.6)$ \\
\hline Orphan-29 & 777737277760771 & & EA & Unknown & Ethiopia_2 & $1(0.6)$ \\
\hline Orphan-30 & 777737337720771 & & EA & Unknown & Haarlem & $1(0.6)$ \\
\hline Orphan-32 & 777737777000731 & मान मान & EA & Unknown & URAL & $2(1.3)$ \\
\hline Orphan-33 & 777777404560771 & 11011001 & EA & Unknown & TUR & $1(0.6)$ \\
\hline Orphan-34 & 777777444760771 & 111 & EA & Unknown & TUR & $3(1.9)$ \\
\hline Orphan-35 & 777777647760701 & 1ान & EA & Unknown & LAM & $1(0.6)$ \\
\hline Orphan-38 & 777777777420571 & पानामाना & EA & Unknown & URAL & $2(1.3)$ \\
\hline Orphan-39 & 401777777420731 & $11011=0$ & EA & Unknown & URAL & $1(0.6)$ \\
\hline Orphan-40 & 603777700017771 & عس سمسرب & EAI & Unknown & Delhi/CAS & $1(0.6)$ \\
\hline Total & & & & & & 37 (23.7) \\
\hline
\end{tabular}

Abbreviations CBN Conformal Bayesian network, EA Euro-American, EAl East-African-Indian, IO IndoOceanic, M. afri., M. africanum

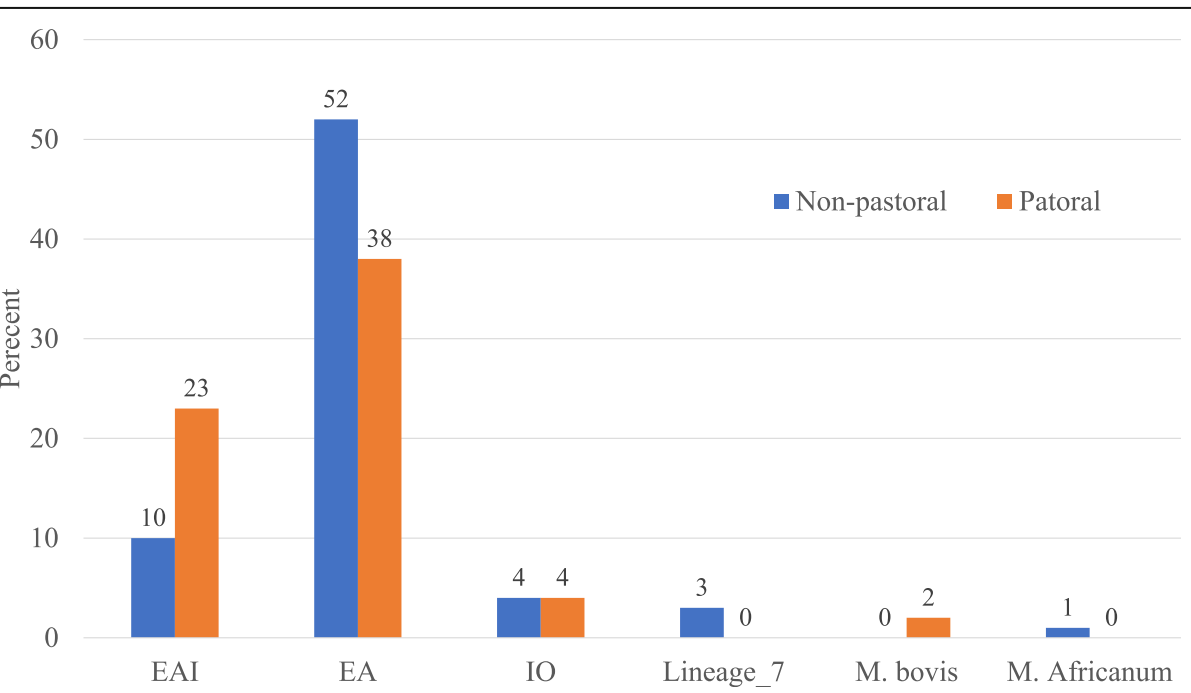

Fig. 1 Major lineage prevalence in pastoral and non-pastoral areas of South Omo, southern Ethiopia. Abbreviations: EA, Euro-American; EAl, EastAfrican-Indian; IO, IndoOceanic; M. afri., M. africanum. The numbers on top of the bars indicate absolute number of Major lineages 
Table 7 Association of different factors with clustering of M. tuberculosis complex

\begin{tabular}{|c|c|c|c|c|c|c|}
\hline Characteristics & Total N (\%) & Clustered N (\%) & COR $(95 \% \mathrm{Cl})$ & $P$ value & AOR $(95 \% \mathrm{Cl})$ & $P$ value \\
\hline \multicolumn{7}{|l|}{ Sex } \\
\hline Female & $64(41.0)$ & $46(71.9)$ & 1.00 & & NA & \\
\hline Male & $92(59.0)$ & $68(73.9)$ & $1.11(0.54-2.27)$ & 0.778 & & \\
\hline \multicolumn{7}{|l|}{ Age in years } \\
\hline $15-29$ & $70(44.9)$ & 55 (78.6) & 1.00 & & NA & \\
\hline $30-44$ & $59(37.8)$ & $41(69.5)$ & $0.62(0.28-1.38)$ & 0.241 & & \\
\hline$>=45$ & $27(17.3)$ & $18(66.7)$ & $0.55(0.20-1.46)$ & 0.227 & & \\
\hline \multicolumn{7}{|l|}{ TB Type } \\
\hline PTB & $130(83.3)$ & $92(70.8)$ & 1.00 & & NA & \\
\hline EPTB & $26(16.7)$ & $22(84.6)$ & $2.27(0.73-7.04)$ & 0.155 & & \\
\hline \multicolumn{7}{|l|}{ Medical history } \\
\hline New cases & $148(94.9)$ & $107(72.3)$ & 1.00 & & NA & \\
\hline Retreat. Cases & $8(5.1)$ & $7(87.5)$ & $2.68(0.32-22.48)$ & 0.363 & & \\
\hline \multicolumn{7}{|l|}{ Residential area } \\
\hline Non-pastoral & $70(51.1)$ & $48(68.6)$ & 1.00 & & 1 & \\
\hline Pastoral & $67(48.9)$ & 56 (83.6) & $2.33(1.03-5.30)$ & 0.043 & $2.29(0.82-6.35)$ & 0.112 \\
\hline \multicolumn{7}{|l|}{ Major lineages ${ }^{\mathrm{a}}$} \\
\hline EAI & 35 (22.9) & $30(85.7)$ & 1.00 & & 1.00 & \\
\hline EA & $105(68.6)$ & $78(74.3)$ & $0.48(0.17-1.37)$ & 0.170 & $0.59(0.16-2.22)$ & 0.439 \\
\hline 10 & $10(6.5)$ & $4(40.0)$ & $0.11(0.02-0.54)$ & $<0.006$ & $0.24(0.03-1.83)$ & 0.169 \\
\hline Lineage_7 & $3(2.0)$ & $2(66.7)$ & $0.33(0.03-4.40)$ & 0.404 & $0.22(0.01-3.50)$ & 0.110 \\
\hline \multicolumn{7}{|l|}{ SIT status } \\
\hline Shared type & $119(76.3)$ & $101(84.9)$ & 1.00 & & 1.00 & \\
\hline Orphan & $37(23.7)$ & $13(35.1)$ & $0.10(0.04-0.22)$ & $<0.001$ & $0.09(0.04-0.25)$ & $<0.001$ \\
\hline \multicolumn{7}{|l|}{ Lineage $^{a}$} \\
\hline Delhi/CAS & 37 (26.6) & $30(81.1)$ & 1.00 & & NA & \\
\hline Haarlem & $22(15.8)$ & $16(72.7)$ & $0.62(0.18-2.17)$ & 0.456 & & \\
\hline URAL & $22(15.8)$ & $15(68.2)$ & $0.50(0.15-1.69)$ & 0.264 & & \\
\hline Ethiopia_2 & $32(23.0)$ & $28(87.5)$ & $1.63(0.43-6.19)$ & 0.470 & & \\
\hline TUR & $10(7.2)$ & $7(70.0)$ & $0.54(0.11-2.65)$ & 0.452 & & \\
\hline Ethiopia_3 & $13(9.4)$ & $11(84.6)$ & $1.28(0.23-7.14)$ & 0.776 & & \\
\hline Ethiopia_1 & $3(2.2)$ & $2(66.7)$ & $0.47(0.04-5.90)$ & 0.556 & & \\
\hline
\end{tabular}

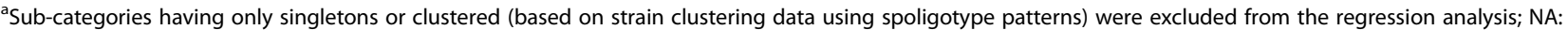
Not applicable; some abbreviations sued here were defined in the legend of Table 2

VNTR are recommended methods for the profiling of MTBC isolates [23, 35]. Both genotyping methods in this study were in range of highly discriminant [27, 34]. MIRU-VNTR has higher discriminatory value than spoligotyping as shown here and in earlier studies [1, 9, 36, 37].

Spoligotyping of South Omo MTBC isolates resulted in a clustering rate of $57.7 \% \%$. This rate agrees with a previous study in Gambella, Southwest Ethiopia [3] which is geographically proximate to the present study site. The rate is lower than a national survey [19] and that of studies in Addis Ababa [26], Northwest Ethiopia [37], Eastern Ethiopia [7], and central Ethiopia [6]. But it was a study in
Western Ethiopia [16]. The MIRU-VNTR clustering rate was $3.9 \%$. The rate is lower than other studies in Ethiopia $[1,9,36,37,43]$ and higher than a Chinese study [15]. Such variability in clustering rate among studies could be due to differences in geography, population density, ethnicity and socio-economic diversity [31]. The low clustering rate in our study could also be associated with low culture recovery rate of samples which make potential isolates from the study population not to be genotyped and/or presence of low TB transmission in South Omo due to geographic expanse which disfavor the transmission as a result of very less crowdedness in the community. 


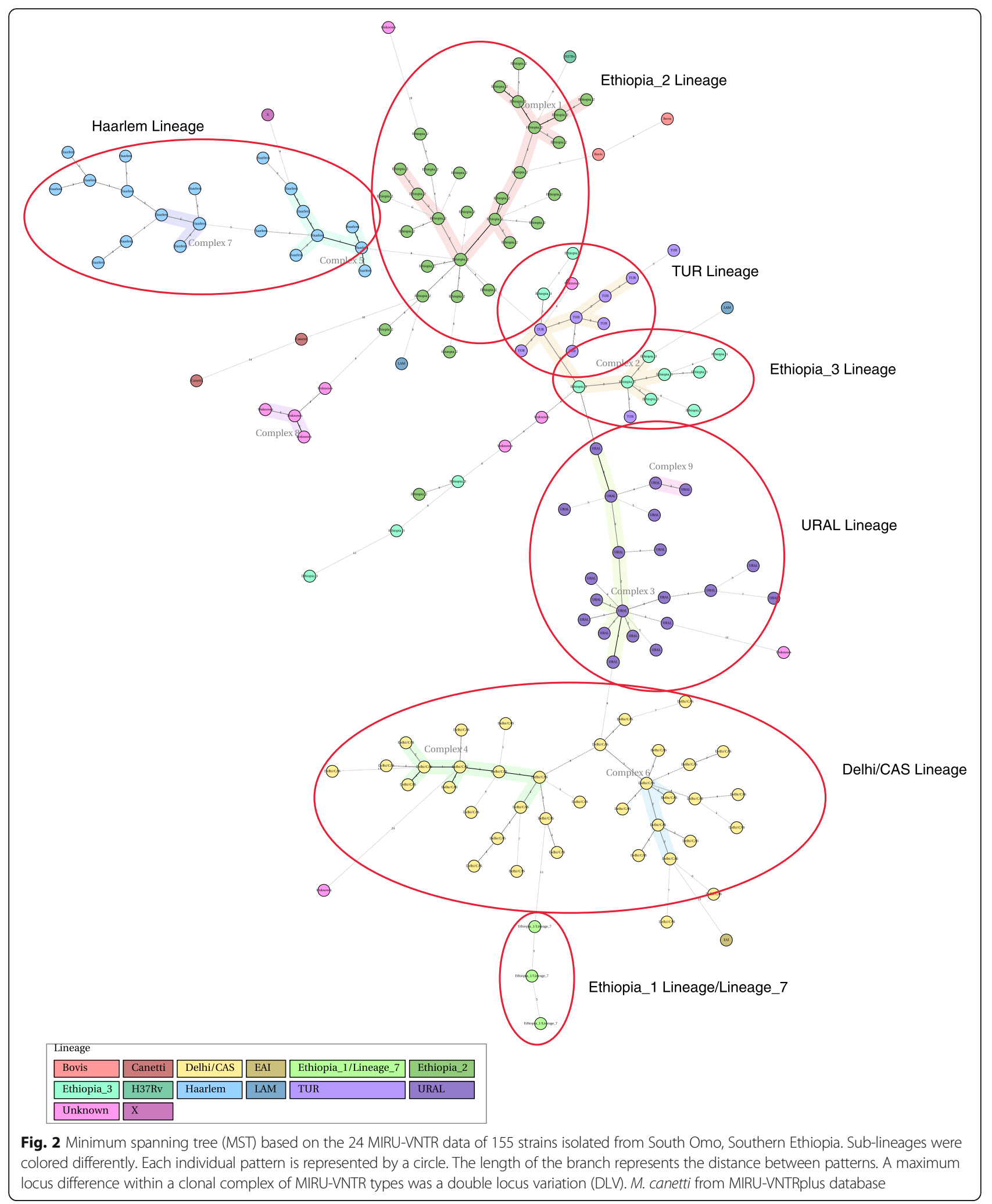

Most MIRU-VNTR alleles in this study were highly and moderately discriminant based on the allelic diversity $(h)$ which is an indirect indicator of the sample representativeness of the study population [34]. Values of $h$ more than 0.8 and less than 0.1 are unsuitable for genotyping [30]. In the present study, all MIRU-VNTR 
loci were suitable for genotyping of the isolates except locus 960 with an $h$ value of 0.810 .

This study identified six major lineages (EA, EAI, IO, lineage_7, M. bovis and $M$. africanum). Four isolates were identified using TB insight database as $M$. africanum which is known to be localized in West Africa. However, three of the four isolates were re-identified using updated version of SITVITWEB as Ethiopian and considered as Lineage_7/Ethiopia_1 $[9,17,37]$ but one isolate remained unknown. The population in the area is endogenous which make the plausibility of an unknown isolate to be $M$. africanum less probable. In general, this might imply the need of updating TB Insight database with newly generated MTBC data from the horn of Africa, particularly Ethiopia.

EA is the most dominant lineage in the world [32], ranging in Ethiopia from 32.5\% near the border to South Sudan [3] to $86.8 \%$ in central Ethiopia [6]. This might highlight the introduction of EA lineages from abroad through the capital city and their expansion to the peripheral areas. In addition, the existence of EA in high number in contrast to Ethiopian lineage, Lineage_7, in the country might indicate the high transmission ability of EA. The contribution of $M$. bovis for TB was low which is supported by data from other studies [7, 17]. While larger clinical studies are needed, our data suggested that the role of $M$. bovis as a causative agent of $\mathrm{TB}$ in pastoral area presumably linked to contact with infected cattle and, consumption of raw milk and meat [5].

According to the updated version of SITVITWEB database, the $\mathrm{T}$ lineage was predominant in this study. This lineage accommodates MTBC strains which do not have phylogeographic specificity [12]. Among the $\mathrm{T}$ sub-lineages, T3 (42.2\%) was the most dominant one in this study. It is also called Ethiopia_2 [9] which is followed by T3-ETH, also called Ethiopia_3 [9]. These isolates supposed to be phylogeographically specific to Ethiopia including well defined Ethiopian lineage also called lineage_7 [17]. The CAS1_Delhi lineage was the second predominant lineage in the study area which was followed by Haarlem. The Haarlem is believed to descend from the European continent [12]. The predominance of the $\mathrm{T}$ lineage in Ethiopia and the CAS lineage in Tanzania [25] and Kenya [29] supports the notion of enrichment of MTBC strains in certain geographies. The Turkey lineage was present in the study area which is believed to be specific to Turkey [45]. This might be associated with presence of Turkey investors in the South Omo. In addition, Ural_1, Manu, Bov, EAI_SOM, and X lineages were identified in less frequency in the area. It is plausible that the observed phylogeographic diversity has linked to considerable international tourism in South Omo, Ethiopia.
When we look at MIRU-VNTRplus based lineage in the present study, Delhi/CAS was the predominat one which is in agreement with previous studies in Ethiopia [9, 36, 37, 43]. Ethiopia_2 is the predominant Ethiopia specific lineage followed by the Ethiopia_3 and lineage 7, similar to a previous study in geographic proximity, Southwestern Ethiopia [36]. But in studies at far distance, in Northwestern Ethiopia [9, 43], the predominant lineage was lineage_7 followed by the Ethiopia_3 and Ethiopia_2 lineages. These findings indicate that the distribution of Ethiopia specific lineages differ moderately from area to area within the country localities. This information is useful for the country's TB Control Program. Almost $6 \%$ of isolates in this study were not assigned into lineages which requires further study and introduction into the genotype database. The relationship among lineages in the MST based on MIRU-VNTR loci was in agreement with similar studies conducted in Ethiopia [9, 37].

Based on the generated data from spoligotyping and MIRU-VNTR, it is possible to say that these two methods can complement each other. But they have different precisions. For instance, MIRU-VNTRplus can identify 37 Delhi/CAS, 22 Haarlem and 22 Ural lineages whereas SITVITWEB can only identify 17 CAS1_Delhi, 16 Haarlem and eight Ural_1. This differences probably associated with the algorithm used by such databases. Finally, from all isolates of South Omo in this study, MIRU-VNTRplus and SITVITWEB didn't assign nine and 56 isolates into lineages, respectively.

The multivariate logistic regression analysis in this study showed none of the variables had association with strain clustering except SIT shared status. Orphan strains were less likely to cluster compared to shared strains which implies that shared strains have higher transmission rate compared to orphan strains in the study area.

We contend that the number of genotyped isolates is sufficient for a primary representation of South Omo's MTBC population structure, assessment of clustering rates and RTIs. However, having less culture recovery rate from samples other than JGH in this study limited our ability to identify clusters and RTIs more comprehensively.

\section{Conclusion}

The MTBC strains derived from TB patients in South Omo were highly diverse while the RTI was low in this marginalized region, as compared to other studies in high TB burden countries. Nonetheless, the genotyping data are useful as an input to map the population structure of MTBC in Ethiopia and to the TB Control Program in the pastoral region. 


\section{Supplementary information}

Supplementary information accompanies this paper at https://doi.org/10. 1186/s12879-020-05394-9.

Additional file 1. Phylogenetic tree for 155 isolates was constructed based on 24 locus MIRU-VNTR. The dendrogram was calculated using neighbor-joining clustering algorithms.

\section{Abbreviations}

AAU: Addis Ababa University; ALIPB: Aklilu Lemma Institute of Pathobiology; AOR: Adjusted odds ratio; Cl: Confidence interval; COR: Crude odds ratio; DOTS: Direct observed treatment short course; EA: Euro-American; EAI: EastAfrican-Indian; EPTB: Extrapulmonary tuberculosis; FNA: Fine needle aspirate; HGDI: Hunter-Gaston Discriminatory Index; JCVI: J. Craig Venter Institute; JRL: Jinka Regional Laboratory; IO: Indo-Oceanic; LJ: Lowenstein Jensen; MIRU-VNTR: Mycobacterial interspersed repetitive unit-variable number of tandem repeat; MTBC: Mycobacterium tuberculosis complex; MST: Minimum spanning tree; PCR: Polymerase chain reaction; PTB: Pulmonary tuberculosis; RD: Region difference; RTI: Recent transmission index; TB: Pulmonary tuberculosis; ZN: Ziehl-Neelsen

\section{Acknowledgments}

The authors would like to thank study participants, South Omo communities and staff members of South Omo Health Offices, Jinka Regional Laboratory, Jinka General Hospital, Health Centers and Health Posts for their valuable support during field sample collection.

\section{Authors' contributions}

BW participated in the study conception, design, data collection, laboratory work, data analysis, data interpretation and manuscript writing. KK participated in its laboratory work, data analysis, data interpretation and manuscript writing. GM participated in its design, data analysis, data interpretation and manuscript writing. $\Pi T$ and FA participated in its data collection and manuscript writing. TM, ST and AZ participated in its laboratory work and manuscript writing. RP and GA participated in the study conception, design, data interpretation and manuscript writing. The authors have read and approved the manuscript.

\section{Funding}

The research was funded by the National Institute of Health (NIH), USA with grant number U01HG007472-01, the Addis Ababa University and the Arba Minch University, Ethiopia. The funders had no role in the study design, data collection, analysis and interpretation, and writing of the manuscript.

\section{Availability of data and materials}

The data generated or analysed during this study are not publicly available due to it may compromise patient anonymity but are available from the corresponding author on reasonable request.

\section{Ethics approval and consent to participate}

The study obtained ethical clearance from ALIPB, AAU Institutional Review Board (ALIPB/IRB/22-B/2012/13) and from the National Research Ethics Committee (Ref No:3.10/785/07). Permission to conduct the study was also obtained from the South Omo Zone Health Department. Written informed consent was obtained from each of the study participants and from their parent or guardian for those who were less than 18 years. Finally, the J. Craig Venter Institutional Review board also approved the study under protocol number 2014-198.

\section{Consent for publication}

$$
\text { Not applicable. }
$$

\section{Competing interests}

The authors declare that they have no competing interests.

\section{Author details}

${ }^{1}$ Aklilu Lemma Institute of Pathobiology, Addis Ababa University, PO. Box 1176, Addis Ababa, Ethiopia. ${ }^{2}$ Department of Biology, Arba Minch University, PO. Box 21, Arba Minch, Ethiopia. ${ }^{3}$ J. Craig Venter Institute, Rockville, MD, USA. ${ }^{4}$ Department of Immunology and Molecular Biology, University of
Gondar, PO. Box 196, Gondar, Ethiopia. ${ }^{5}$ Ethiopian Public Health Institute, P.O.Box 1242, Addis Ababa, Ethiopia. ${ }^{6} J i n k a$ General Hospital, Jinka, Ethiopia ${ }^{7}$ Department of General Surgery, College of Medicine and Health Sciences, University of Gondar, Gondar, Ethiopia. ${ }^{8}$ Department of Veterinary Medicine, College of Food and Agriculture, United Arab Emirates University, P.O.Box 15551, Al Ain, UAE.

Received: 3 May 2020 Accepted: 2 September 2020

Published online: 13 October 2020

\section{References}

1. Ali S, Beckert P, Haileamlak A, Wieser A, Pritsch M, Heinrich N, et al. Drug resistance and population structure of $M$ tuberculosis isolates from prisons and communities in Ethiopia. BMC Infect Dis. 2016;16:687.

2. Allix-Beguec C, Harmsen D, Weniger T, Supply P, Niemann S. Evaluation and strategy for use of MIRU-VNTRplus, a multifunctional database for online analysis of genotyping data and phylogenetic identification of Mycobacterium tuberculosis complex isolates. J Clin Microbiol. 2008;46:2692-9.

3. Asebe G, Worku A, Zewude A, Tafess K, Ameni G. Molecular characterization of Mycobacterium tuberculosis complex in Gambella region, Southwest Ethiopia. Afr J Microbiol Res. 2016;10:8.

4. AU. Policy Framework for Pastoralism in Africa: Securing, Protecting and Improving the Lives, Livelihoods and Rights of Pastoralist Communities Addis Ababa. Ethiopia: Africa Union; 2010.

5. Ayele WY, Neill SD, Zinsstag J, Weiss MG, Pavlik I. Bovine tuberculosis: an old disease but a new threat to Africa. Int J Tuberculosis Lung Dis. 2004:8:924-37.

6. Bedewi Z, Worku A, Mekonnen Y, Yimer G, Medhin G, Mamo G, et al. Molecular typing of Mycobacterium tuberculosis complex isolated from pulmonary tuberculosis patients in Central Ethiopia. BMC Infect Dis. 2017;17:184.

7. Belay M, Ameni G, Bjune G, Couvin D, Rastogi N, Abebe F. Strain diversity of Mycobacterium tuberculosis isolates from pulmonary tuberculosis patients in Afar pastoral region of Ethiopia. Biomed Res Int. 2014;2014 https://doi.org/ $10.1155 / 2014 / 238532$.

8. Berg S, Schelling E, Hailu E, Firdessa R, Gumi B, Erenso G, et al. Investigation of the high rates of extrapulmonary tuberculosis in Ethiopia reveals no single driving factor and minimal evidence for zoonotic transmission of Mycobacterium bovis infection. BMC Infect Dis. 2015;15:112

9. Biadglegne F, Merker M, Sack U, Rodloff AC, Niemann S. Tuberculous lymphadenitis in Ethiopia predominantly caused by strains belonging to the Delhi/CAS lineage and newly identified Ethiopian clades of the Mycobacterium tuberculosis complex. PLoS One. 2015;10:e0137865.

10. Bifani PJ, Mathema B, Liu Z, Moghazeh S, Shopsin B, Tempalski B, et al. Identification of a w variant outbreak of Mycobacterium tuberculosis via population-based molecular epidemiology. JAMA. 1999;282:2321-7.

11. Brosch R, Gordon SV, Marmiesse M, Brodin P, Buchrieser C, Eiglmeier K, et al. A new evolutionary scenario for the Mycobacterium tuberculosis complex. Proc Natl Acad Sci U S A. 2002;99:3684-9.

12. Brudey K, Driscoll JR, Rigouts L, Prodinger WM, Gori A, Al-Hajoj SA, et al. Mycobacterium tuberculosis complex genetic diversity: mining the fourth international spoligotyping database (SpolDB4) for classification, population genetics and epidemiology. BMC Microbiol. 2006;6:23.

13. Couvin D, David A, Zozio T, Rastogi N. Macro-geographical specificities of the prevailing tuberculosis epidemic as seen through SITVIT2, an updated version of the Mycobacterium tuberculosis genotyping database. Infect Genet Evol. 2019;72:31-43 PMID: 30593925.

14. CSA. Summary and statistical report of the 2007 population and housing census- population size by age and sex: Federal Democratic Republic of Ethiopia, population census commission, with support from UNFPA; 2008.

15. Dai GM, Zhang ZG, Ding PJ, Zhang Q, Wang L, Wang LX, et al. Differences in the population of genetics of Mycobacterium tuberculosis between urban migrants and local residents in Beijing, China. Chin Med J. 2013;126: 4066-71.

16. Disassa H, Tafess K, Worku A, Ameni G. A preliminary study on molecular characterization of Mycobacterium tuberculosis in Benishangul Gumuz region, Western Ethiopia. B M R J. 2015;10:1-10.

17. Firdessa R, Berg S, Hailu E, Schelling E, Gumi B, Erenso G, et al. Mycobacterial lineages causing pulmonary and extrapulmonary tuberculosis, Ethiopia. Emerg Infect Dis. 2013;19:460-3.

18. $\mathrm{FMOH}$. Guidelines for clinical and programmatic management of TB, TB/HIV and leprosy in Ethiopia. Addis Ababa: FMOH; 2013. 
19. Getahun M, Ameni G, Kebede A, Yaregal Z, Hailu E, Medihn G, et al. Molecular typing and drug sensitivity testing of Mycobacterium tuberculosis isolated by a community-based survey in Ethiopia. BMC Public Health. 2015; 15:751.

20. Hermans PWM, Messadi F, Guebrexabher H, Van Soolingen D, De Haas PEW, Heersma $\mathrm{H}$, et al. Analysis of the population structure of Mycobacterium tuberculosis in Ethiopia, Tunisia, and the Netherlands: usefulness of DNA typing for global tuberculosis epidemiology. J Infect Dis. 1995;171:1504-13.

21. Hunter PR, Gaston MA. Numerical index of the discriminatory ability of typing systems: an application of Simpson's index of diversity. J Clin Microbiol. 1988;26:2465-6.

22. Jagielski T, Minias A, Van Ingen J, Rastogi N, Brzostek A, Zaczek A, et al. Methodological and clinical aspects of the molecular epidemiology of Mycobacterium tuberculosis and other mycobacteria. Clin Microbiol Rev. 2016:29:239-90

23. Kamerbeek J, Schouls L, Kolk A, Van Agterveld M, Van Soolingen D, Kuijper S, et al. Simultaneous detection and strain differentiation of Mycobacterium tuberculosis for diagnosis and epidemiology. J Clin Microbiol. 1997;35:907-14.

24. Kebede AH, Alebachew Z, Tsegaye F, Lemma E, Abebe A, Agonafir M, et al. The first population-based national tuberculosis prevalence survey in Ethiopia, 2010-2011. Int J Tuberc Lung Dis. 2014;18:635-9.

25. Mbugi EV, Katale BZ, Siame KK, Keyyu JD, Kendall SL, Dockrell HM, et al. Genetic diversity of Mycobacterium tuberculosis isolated from tuberculosis patients in the Serengeti ecosystem in Tanzania. Tuberculosis. 2015;95:170-8.

26. Mihret A, Bekele Y, Loxton AG, Jordan AM, Yamuah L, Aseffa A, et al. Diversity of Mycobacterium tuberculosis isolates from new pulmonary tuberculosis cases in Addis Ababa, Ethiopia. Tuberc Res Treat. 2012;2012: 892079

27. Mokrousov I. Revisiting the Hunter Gaston discriminatory index: note of caution and courses of change. Tuberculosis. 2017;104:20-3.

28. Nebenzahl-Guimaraes H, Yimer SA, Holm-Hansen C, De Beer J, Brosch R, Van Soolingen D. Genomic characterization of Mycobacterium tuberculosis lineage 7 and a proposed name: 'Aethiops vetus'. Microbial Genomics. 2016; 2:e000063

29. Ogaro TD, Githui W, Kikuvi G, Okari J, Asiko V, Wangui E, et al. Diversity of Mycobacterium tuberculosis strains in Nairobi, Kenya. Afr J Health Sci. 2012;20:9.

30. Pan XL, Zhang CL, Nakajima C, Fu J, Shao CX, Zhao LN, et al. A quantitative and efficient approach to select MIRU-VNTR loci based on accumulation of the percentage differences of strains for discriminating divergent Mycobacterium tuberculosis sublineages. Emerg Microbes Infect. 2017;e68:6.

31. Pareek M, Evans J, Innes J, Smith G, Hingley-Wilson S, Lougheed KE, et al. Ethnicity and mycobacterial lineage as determinants of tuberculosis disease phenotype. Thorax. 2013;68:221-9.

32. Reed MB, Pichler VK, Mcintosh F, Mattia A, Fallow A, Masala S, et al. Major Mycobacterium tuberculosis lineages associate with patient country of origin. J Clin Microbiol. 2009;47:1119-28.

33. Small PM, Hopewell PC, Singh SP, Paz A, Parsonnet J, Ruston DC, et al. The epidemiology of tuberculosis in San Francisco. A population-based study using conventional and molecular methods. N Engl J Med. 1994;330:1703-9.

34. Sola C, Filliol I, Legrand E, Lesjean S, Locht C, Supply P, et al. Genotyping of the Mycobacterium tuberculosis complex using MIRUs: association with VNTR and spoligotyping for molecular epidemiology and evolutionary genetics. Infect Genet Evol. 2003;3:125-33.

35. Supply P, Allix C, Lesjean S, Cardoso-Oelemann M, Rusch-Gerdes S, Willery E, et al. Proposal for standardization of optimized mycobacterial interspersed repetitive unit-variable-number tandem repeat typing of Mycobacterium tuberculosis. J Clin Microbiol. 2006:44:4498-510.

36. Tadesse M, Abebe G, Bekele A, Bezabih M, De Rijk P, Meehan CJ, et al. The predominance of Ethiopian specific Mycobacterium tuberculosis families and minimal contribution of Mycobacterium bovis in tuberculous lymphadenitis patients in Southwest Ethiopia. Infect Genet Evol. 2017;55:251-9.

37. Tessema B, Beer J, Merker M, Emmrich F, Sack U, Rodloff AC, et al. Molecular epidemiology and transmission dynamics of Mycobacterium tuberculosis in Northwest Ethiopia: new phylogenetic lineages found in Northwest Ethiopia. BMC Infect Dis. 2013;13:131.

38. Weniger T, Krawczyk J, Supply P, Niemann S, Harmsen D. MIRU-VNTRplus: a web tool for polyphasic genotyping of Mycobacterium tuberculosis complex bacteria. Nucleic Acids Res. 2010;38:W326-31.

39. WHO. Global tuberculosis report; 2019. https://www.who.int/tb/publications/ global_report/en/, 2019. Accessed 6 Apr 2020
40. Wilton S, Cousins D. Detection and identification of multiple mycobacterial pathogens by DNA amplification in a single tube. PCR Methods Appl. 1992; 1:269-73.

41. Wondale B, Medihn G, Teklu T, Mersha W, Tamirat M, Ameni G. A retrospective study on tuberculosis treatment outcomes at Jinka general hospital, southern Ethiopia. BMC Res Notes. 2017;10:680.

42. Yimer SA, Birhanu AG, Kalayou S, Riaz T, Zegeye ED, Beyene GT, et al. Comparative proteomic analysis of Mycobacterium tuberculosis lineage 7 and lineage 4 strains reveals differentially abundant proteins linked to slow growth and virulence. Front Microbiol. 2017;8:795.

43. Yimer SA, Norheim G, Namouchi A, Zegeye ED, Kinander W, Tonjum T, et al. Mycobacterium tuberculosis lineage 7 strains are associated with prolonged patient delay in seeking treatment for pulmonary tuberculosis in Amhara region, Ethiopia. J Clin Microbiol. 2015;53:1301-9.

44. Zhou Y, Van Den Hof S, Wang S, Pang Y, Zhao B, Xia H, et al. Association between genotype and drug resistance profiles of Mycobacterium tuberculosis strains circulating in China in a national drug resistance survey. PLoS One. 2017;12:e0174197.

45. Zozio T, Allix C, Gunal S, Saribas Z, Alp A, Durmaz R, et al. Genotyping of Mycobacterium tuberculosis clinical isolates in two cities of Turkey: description of a new family of genotypes that is phylogeographically specific for Asia minor. BMC Microbiol. 2005:5:44.

46. Meehan CJ, Moris P, Kohl TA, Pečerska J, Akter S, Merker M, et al. The relationship between transmission time and clustering methods in Mycobacterium tuberculosis epidemiology. EBioMedicine. 2018;37:410-6.

47. Tilahun M, Ameni G, Desta K, Zewude A, Yamuah L, Abebe M, Aseffa A. Molecular epidemiology and drug sensitivity pattern of Mycobacterium tuberculosis strains isolated from pulmonary tuberculosis patients in and around Ambo Town, Central Ethiopia. PLoS One. 2018;13(2):e0193083.

48. Bruchfeld J, Aderaye G, Palme IB, Bjorvatn B, Britton S, Feleke Y, et al. Evaluation of Outpatients with Suspected Pulmonary Tuberculosis in a High HIV Prevalence Setting in Ethiopia: Clinical, Diagnostic and Epidemiological Characteristics. Scand J Infect Dis. 2002:34:331-7.

\section{Publisher's Note}

Springer Nature remains neutral with regard to jurisdictional claims in published maps and institutional affiliations.

Ready to submit your research? Choose BMC and benefit from:

- fast, convenient online submission

- thorough peer review by experienced researchers in your field

- rapid publication on acceptance

- support for research data, including large and complex data types

- gold Open Access which fosters wider collaboration and increased citations

- maximum visibility for your research: over $100 \mathrm{M}$ website views per year

At $\mathrm{BMC}$, research is always in progress.

Learn more biomedcentral.com/submission 